Shanaya Rathod, Crisis Resolution Home Treatment Service, Mid Hampshire and Eastleigh Locality, Melbury Lodge, Romsey Road, Winchester SO22 5DG, UK. Email: shanayarathod@nhs.net: Farooq Naeem, Peter Phiri, David Kingdon, Mental Health Group, University Department of Psychiatry, Royal South Hants Hospital, Southampton, UK

doi: 10.1192/bjp.193.3.256a

Author's reply: Mushtaq shares my concern about inappropriate medicalisation, but sees short-term interventions such as CBT as something apart. I must disagree: talk therapies delivered in the National Health Service by mental health professionals are part and parcel of what profession and public understands by 'medical'.

In working to produce 'culturally sensitive CBT' for depression in Pakistan, Rathod et al hope that mere adaptation of standard practices and manuals, and good translations, will do the trick. I'm afraid I challenge the assumption that Western psychiatric templates can generate a universally valid knowledge base. ${ }^{1}$ Methodologies routinely fail the core test of scientific validity, which relates to the 'nature of reality' for the individuals under study.

Globalising Western psychiatric approaches is not value free. A telling example of the moral and political shifts to which I alluded in the debate is provided by the invasion of Latvia by the diagnosis of depression. ${ }^{2}$ This was prompted by the translation of ICD into Latvian, and by conferences organised by pharmaceutical companies to educate psychiatrists and general practitioners (who in turn educated their patients) about this new diagnostic category. This was a radical departure from the traditional language of (largely somatic) distress - notably nervi - shared by doctors and lay public. To present nervi was to invite a life story, which could include a critical commentary on disorder or dysfunction outside the self, in wider society and politics. The doctor-mediated shift from nervi to depression is a shift away from the lived contexts that nervi embodied, the focus now inwards to the individual person. With this comes the internalisation of a heightened sense of personal accountability for life circumstances. However, at the same time post-Soviet Latvian society has lost much of its former sense of stability and security, and most people have in fact less control over their lives. The narrative structure of these new accounts of distress indicates that Latvians have internalised the values of capitalist enterprise culture and the responsibility for personal failure that goes with it. It is this shaping of a different kind of citizen that is evoked in the globalisation of depression.

1 Summerfield D. How scientifically valid is the knowledge base of global mental health? BMJ 2008; 336: 992-4.

2 Skultans V. From damaged nerves to masked depression: inevitability and hope in Latvian psychiatric narratives. Soc Sci Med 2003; 56: 2421-31.

Derek Summerfield, Institute of Psychiatry, King's College London, UK. Email: derek.summerfield@slam.nhs.uk

doi: 10.1192/bjp.193.3.257

\section{Integrated multidisciplinary diagnostic approach for dementia}

Wolfs et al have described a cluster randomised controlled trial in The Netherlands in which patients with suspected dementia received integrated multidisciplinary assessment or usual care. ${ }^{1}$ Input to the intervention group aimed to combine the hospitalbased approach of a memory clinic with the care-oriented approach of a community mental health team. This intervention led to some modest improvements in outcome. Usual care during the trial was provided by the general practitioner, or involved referral to a regional memory clinic, a department of geriatric medicine or mental health service for the elderly.

The integrated approach only lasted for about 2 weeks, after which detailed diagnostic and therapeutic advice was given to general practitioners. Given that dementia is a progressive neurodegenerative disorder with constantly changing medical and social care needs, we would be surprised if this intervention could sustain superiority over ongoing care from any community mental health service for elderly people - no matter how rudimentary. Further details on treatment as usual would have been useful, as would a reanalysis of the results taking into account the different types of service received by the control patients.

We agree with Wolf et al that memory clinics need to integrate with multidisciplinary community services. We have argued previously that the sub-specialist memory clinics in the UK have not been useful in the overall management of dementia since they have distorted care priorities and have focused on the prescribing and monitoring of medication. ${ }^{2}$ Wolf $e t$ al's controlled trial has provided support for integration of services for the diagnosis and care of dementia. This has to be organised not only in the initial diagnostic stages but also on an ongoing basis, with close liaison between multidisciplinary health services, local social work departments and primary care throughout the course of patients' progressive illness.

1 Wolfs CAG, Kessels A, Dirksen CD, Severens JL, Verhey FRJ. Integrated multidisciplinary diagnostic approach for dementia care: randomised controlled trial. Br J Psychiatry 2008; 192: 300-5.

2 Pelosi AJ, McNulty S, Jackson G. Role of cholinesterase inhibitors in dementia needs rethinking. $B M J$ 2006; 333: 491-3.

Seamus V. McNulty, North West Kilmarnock Area Centre, Western Road, Kilmarnock KA3 1NQ, UK. Email: seamus.mculty@aapct.scot.nhs.uk; Graham A. Jackson, Leverndale Hospital, Glasgow; Anthony J. Pelosi, Hairmyres Hospital, East Kilbride, UK

doi: 10.1192/bjp.193.3.257a

Authors' reply: Organisational models designed to create connectivity, alignment and collaboration within and between the cure and care sectors are needed, and our study provides the evidence to support this approach. Our diagnostic intervention indeed lasted only a few weeks, but in our view, dementia care is a chain of services, starting with a short but comprehensive diagnostic phase resulting in a treatment plan that lasts throughout the course of the illness. Our intervention was merely the beginning of that chain, and we acknowledge that this is an ongoing process.

In contrast to McNulty et al, who found the results of our study modest, we value a difference of almost $10 \%$ between groups regarding health-related quality of life as substantial and clinically relevant, and higher than found in any pharmacological study in dementia so far.

The suggestion of McNulty et al to compare different types of services would be interesting, but the design of our study was not appropriate for such a reanalysis, as it would be subject to confounding by indication.

Nevertheless, McNulty et al raise the important point that dementia care needs an integrated approach on an ongoing basis, and we agree wholeheartedly.

Frans R. Verhey, Department of Psychiatry \& Neuropsychology, Maastricht University, Maastricht, The Netherlands. Email: f.verhey@np.unimaas.nl;

Claire A. Wolfs, Department of Psychiatry \& Neuropsychology, Maastricht University; Alfons Kessels, Carmen D. Dirksen, Department of Clinical Epidemiology and Medical Technology Assessment, University Hospital, Maastricht; Johan L. Severens, Department of Health Organisation, Policy \& Economics, Maastricht University,

The Netherlands

doi: 10.1192/bjp.193.3.257b 